\title{
ON COMMUTATIVE PRIME POWER SUBGROUPS OF THE NORM ${ }^{1}$
}

\author{
BY \\ LAWRENCE Thomas Wos
}

The norm $N(G)$ of a group $G$ is the set of elements $x \in G$ such that $x+T=$ $T+x$ for every subgroup $T$ of $G$. ( $G$ will be written additively in spite of the fact that $G$ need not be commutative.) The norm is, therefore, the intersection of the normalizers of all the subgroups of $G$ and is a characteristic, hence normal, subgroup of $G$. The main results of the paper are that the norm $N(G)$ is contained in the third center of $G$, and that the group of automorphisms induced on $N(G)$ by $G$ is nilpotent of class 2 . With certain reservations concerning the prime 2 , we prove furthermore that the norm is contained in the second center if and only if the group of automorphisms induced on $N(G)$ by $G$ is commutative.

R. Baer [4] proved the result that the norm of a group is 0 if and only if the center is 0 . This theorem follows directly from our result that the norm is contained in the third center, for if the center is 0 , then the third center is 0 , and, a fortiori, the norm is 0 ; the necessity follows from the obvious fact that the norm contains the center.

The main results of the paper are obtained from a larger program, namely, the consideration of norm pairs $P, S$. A norm pair $P, S$ is defined as a pair of groups $P$ and $S$, where $P$ is a commutative $p$-group and a normal subgroup of a group $G$ and contained in the norm of $G$, and where $S$ is the group of automorphisms induced on $P$ by $G$. If $a$ is an automorphism of the group $P$, let $F(a)$ denote the set of $x \in P$ such that $x=x a$, i.e., the elements left fixed by $a$; let $P(1-a)$ denote the set of $x-x a=x(1-a)$ for $x \in P$.

A pair of groups $P, S$ is termed a norm-like pair if $P$ is a commutative $p$ group and $S$ is a group of automorphisms of $P$ such that, for every $a \in S$, $P(1-a)$ is cyclic and contained in $F(a)$. We prove that norm pairs are norm-like and that norm-like pairs, under certain conditions, are norm pairs. (If $P, S$ is a norm-like pair, then composition in $P$ will be denoted as addition whereas composition in $S$ will be multiplication.)

If $P, S$ is a norm-like pair, the elements $a \epsilon S$ of $S$ satisfy the equality, $k(1-a)=1-a^{k}$ for all integers $k$. With the aid of this equality it will be shown that, if $P, S$ is a norm-like pair, then $S$ is a $p$-group and, in fact, the order of $a \epsilon S$ equals the order of $P(1-a)$. For such pairs $P, S$ we prove $S$ is of class 2 and $P=F_{3}$, where $F_{0}=0$ and $F_{i}=$ the set of $x \in P$ such that $x-x a \in F_{i-1}$ for every $a \in S$ with $i=1, \cdots$; with $p \neq 2, S$ is commutative if and only if $P=F_{2}$. These results demonstrate the strong connection between norm pairs and norm-like pairs, especially when one realizes that $F_{i}$

Received August 22, 1957.

1 Work performed under the auspices of the U. S. Atomic Energy Commission. 
plays the role of the intersection of $P$ with the $i^{\text {th }}$ member of the ascending central series.

\section{Notations}

$N(G)=$ the norm of the group $G$.

$Z(G)=$ the center of $G$.

$Z_{i}(G)=$ the $i^{\text {th }}$ member of the ascending central series of $G$; in particular, $Z_{1}(G)=Z(G)$.

$G_{p}=$ the $p$-component of $G$, i.e, the set of all elements of $G$ whose order is a power of the prime $p$.

$o(x)=$ the order of the element $x$.

$o(G)=$ the order of the group $G$.

$(a)=$ subgroup generated by $a$.

\section{Section 1}

Throughout the whole of the paper $P$ will denote an additive commutative $p$-group, i.e., a group all of whose elements have order a power of some fixed prime $p$. If $P$ is contained in some larger group $G$ as a normal subgroup and $a$ is an element of $G$, we shall identify $a$ with the automorphism $a$ induces on $P$ and denote the automorphism by $a$. Consequently $P(1-a)$ is, for $a \epsilon G$, the commutator subgroup of the group generated by $a$ and $P ; F(a)$ is the centralizer of $a$ in $G$. Since $P$ is commutative, $P(1-a)$ and $F(a)$ are clearly subgroups of $P$ for every automorphism $a$ of $P$.

Throughout this section assume that $P$ is contained in the norm $N(G)$ of a group $G$ and that $P$ is a normal subgroup of $G$. Thus $G$ induces an automorphism group $S$ on $P$ and $P, S$ is, therefore, a norm pair.

Lemma 1.1. The centralizer of $P$ in $G$ contains all elements of infinite order or of order prime to $p$.

Proof. Consider $w \in G$ such that $o(w)=0$ or $o(w)$ is prime to $p$. $(w) \cap P=0$ since $P$ is a $p$-group. For $x \in P$

$$
\begin{aligned}
{[w, x] } & =-w-x+w+x=(-w-x+w)+x \in P \text { since } P \text { is normal in } G, \\
& =-w+(-x+w+x) \epsilon(w) \text { since } x \in P \leqq N(G) .
\end{aligned}
$$

So $[w, x] \epsilon(w) \cap P=0$, or $w+x=x+w$ for every $x \in P$, and the proof is complete.

LEMMA 1.2. If $a$ is an automorphism induced by an element of $G$ on the p-group $P$, then $o(a)$ is a power of $p$, and there exists a p-element $a \in G$ inducing the automorphism $a$.

Proof. If $a=1$, there is nothing to prove. Consider $a \neq 1$. There exists a $w \in G$ such that $w$ induces $a$ on $P$. If $o(w)=0$ or is prime to $p, w$ commutes with $P$ elementwise, which contradicts $a \neq 1$; therefore, $o(w)=p^{k} q$ with $0<k$ and $q$ prime to $p$. So there exist elements $a$ and $\tilde{w}$ in $G$ such that 
$w=a+\tilde{w}$ and such that $o(a)=p^{k}$ and $o(\tilde{w})=q . \quad \tilde{w}$ commutes with $P$, so $a \epsilon G$ induces the automorphism $a$ on $P$. The order of the automorphism $a$ is the smallest multiple of $a \epsilon G$ contained in the centralizer $C$ of $P$ in $G$, which is the smallest multiple of $a$ contained in $C \cap(a)$, which equals the index of $C \cap(a)$ in $(a)$ since $(a)$ is a cyclic group; but this index is a power of $p$ since $a \epsilon G$ is a $p$-element.

Theorem 1.1. Let $P, S$ be a norm pair; then, $P(1-a)$ is, for every a $\epsilon S$, a cyclic group contained in $F(a)$ and isomorphic to $P / F(a)$; if $P$ is not contained in the center $Z(G)$ of $G$, then $P$ has bounded order, i.e., there exists a positive integer $m$ such that $p^{m} P=0$.

Proof. Let $a$ be an element of $S$. The mapping of $x \in P$ to $x(1-a)=$ $x-x a$ is an endomorphism of $P$ with kernel $F(a)$ and image $P(1-a)$. By the first isomorphism theorem, $P(1-a)$ is, therefore, isomorphic to $P / F(a)$. Let $a \in G$ induce the automorphism $a$. For every $x \in P$ we have $x-a-x \in(a)$ since $P$ is part of the norm of $G$; therefore, $P(1-a) \leqq(a)$ and thus is a cyclic group which is contained in $F(a)$ since $a$ commutes with multiples of itself.

Now assume that $P$ is not contained in $Z(G)$. Then there exists an $a \in S$ with $a \neq 1$. Let $a \epsilon G$ be a $p$-element inducing $a$ on $P$; such an element exists by Lemma 1.2. By the above argument, $P(1-a)$ is contained in $(a)$ and is cyclic. Since $P$ is a $p$-group, $P(1-a)$ has order $p^{i}$ for some positive integer $i$ and, therefore, equals $p^{k-i} a$ where the order of $a \epsilon G$ is $p^{k}$. Consider $x \in F(a)$. Since $P$ is commutative, $a+x$ induces $a$ on $P . P(1-a) \leqq$ $(a+x)$, so there exists an integer $j$ such that $p^{k-i} a=j(a+x)=j a+j x$. Now we distinguish two cases.

Case 1. $(x) \cap(a)=0$.

Therefore, $j x=p^{k-i} a-j a=0$, or $j a=p^{k-i} a . \quad j a=p^{k-i} a \neq 0$. Hence $j=p^{k-i} j^{\prime}$, where $j^{\prime} \equiv 1 \bmod p^{i}$. Furthermore $j x=0$; and this implies $p^{k-i} x=0$, since $o(x)$ is a power of $p$. Hence $o(x)$ divides $p^{k-i}$.

Case 2. $(x) \cap(a) \neq 0$.

$(x) \cap(a)$ therefore contains all elements of order $p$ in $(a)$, which are in turn all elements of order $p$ in $(x)$. Let $U$ be the subgroup of $G$ generated by $a$ and $x$; $U$ is a finite commutative $p$-group since $x \in F(a)$ and $x$ and $a$ are $p$-elements. The maximum of $o(u)$ for $u \in U$ equals the maximum of $o(x)$ and $o(a)$ since $x$ and $a$ are $p$-elements for the same prime $p$. Assume by way of contradiction that the maximum order of the elements in $U$ is $o(x)$. Since every element of maximum order in a finite commutative group generates a direct summand, $(x)$ is a direct summand of $U$. Hence there exists a subgroup $V$ such that $U=(x)+V$ with $(x) \cap V=0$. If $V \cap(a) \neq 0, V$ contains all elements of order $p$ in $(a)$; but these elements are in $(x)$, which contradicts $V \cap(x)=0$. So $V \cap(a)=0$. Since $U$ is generated by $a$ and $x$, the direct summand $V$ is cyclic, and there exists $v=h a+j x$ for integers $j$ and $h$ such that $V=(v)$. Then $P(1-v)=P(1-h a) \leqq V \cap(h a) \leqq V \cap(a)=0$, so that $v$ induces 1 
on $P$. But $a$ is a linear combination of $v$ and $x$, both of which commute with $P$, and $a$ does not induce the identity on $P$. Thus we have a contradiction, and our assumption that $o(x)$ was maximal in $U$ is false. So $o(x) \leqq o(a)$, and this holds for all $x \in F(a)$ since $x$ was chosen arbitrarily. As noted before, $P / F(a)$ is isomorphic to $P(1-a)$. So $P / F(a)$ has order $p^{i}$, or $p^{i} y \epsilon F(a)$ for every $y \in P$. But $p^{k} x=0$ for every $x \in F(a)$ from the above; therefore $p^{k+i} P=0$, and $P$ has bounded order.

\section{Section II}

We now turn to more general considerations and therefore make the following definition.

Definition. We term the pair $P, S$ of groups $P$ and $S$ a norm-like pair if $S$ is an automorphism group of $P$ such that, for every $a \in S$,

1. $P(1-a)$ is cyclic, and,

2. $P(1-a)$ is contained in $F(a)$.

Throughout this section $P, S$ will be a norm-like pair, but $P$ is not assumed to be contained in some larger group. However, we assume that $P$ is a $p$ group. We shall use the additive notation in $P$, but the multiplicative notation in $S$.

Lemma 2.1. For every pair of automorphisms $a$ and $b$ in $S, F(a) b=F(a)$.

Proof. Let $x$ be an arbitrary element of $F(a)$. Then

$$
x(1-b)=x-x b=x-x a b=x(1-a b) .
$$

From property 2 of $S$,

$$
y=x(1-b)=x(1-a b) \epsilon F(b) \cap F(a b) ;
$$

so $y=y b=y a b$. By applying $b^{-1}, y=y a$. Since $x \in F(a), x-y=$ $x b \in F(a)$. So $F(a) b \leqq F(a)$. By the same argument but with $b$ replaced by $b^{-1}, F(a) b^{-1} \leqq F(a)$. Applying $b$ to the last inequality, $F(a) \leqq F(a) b$, which completes the proof.

The following lemma proves a very useful and somewhat surprising equality obeyed by the elements of $S$.

Lemma 2.2. The following three properties of elements $a$ and $b$ in $S$ are equivalent: $P(1-a) \leqq F(b) ;(1-a) b=1-a ; 1-a b=(1-a)+(1-b)$.

Proof. The equivalence of the first two properties, and their equivalence with the third property, may be inferred from the identity:

$$
1-a b=1-b+b-a b=(1-b)+(1-a) b .
$$

Corollary 2.1. If $P(1-a) \leqq F(b)$ and $P(1-b) \leqq F(a)$, then $a b=b a$. 
Proof. The hypotheses imply by Lemma 2.2 that

$$
1-a b=(1-a)+(1-b)=1-b a ;
$$

and this in turn implies $a b=b a$.

Corollary 2.2. If $U$ is a subgroup of $S$ such that $P(1-a) \leqq F(b)$ for every pair of elements $a, b$ in $U$, then mapping $x$ in $U$ on $1-x$ (in the ring of endomorphisms of $P$ ) is a homomorphism.

This is an immediate consequence of Lemma 2.2.

Corollary 2.2'. If $a$ is an element in $S$ and $k$ an integer, then $1-a^{k}=k(1-a)$.

This is a special case of Corollary 2.2.

Lemma 2.3. For every $a \epsilon S, o(a)=o(P(1-a))$.

Proof. From property 1 of $S$, there exists an $x \in P$ such that $x(1-a)$ generates $P(1-a)$. Let $o(P(1-a))=r$. If $y \in P$, then

$$
\begin{array}{rlr}
y a^{r} & =y-y+y a^{r}=y-y\left(1-a^{r}\right)=y-r y(1-a) \quad \text { by Corollary 2.2', } \\
& =y & \text { since } r=o(P(1-a)) .
\end{array}
$$

On the other hand $x a^{i}=x-i x(1-a)$, so that $x=x a^{i}$ if, and only if, $r \mid i$. Hence $o(a)=r=o(P(1-a))$.

Since $P$ is assumed to be a $p$-group for the prime $p, S$ is therefore a $p$-group for that prime.

Lemma 2.4. If, for $a$ and $b$ in $S, C$ is the subgroup of $S$ generated by $a$ and $b$, and if $P(1-C)$ is the subgroup of $P$ generated by all the $P(1-c)$ for $c \epsilon C$, then $P(1-C)=P(1-a)+P(1-b)$, and $o(c) \leqq \max [o(a), o(b)]$ for every $c \in C$.

Proof. It is clear that $Q=P(1-a)+P(1-b) \leqq P(1-C)$. Since $x(1-a) b=x(1-a)+x a(1-b)-x(1-b)$, it follows that $Q b \leqq Q$. Likewise we see that $Q a \leqq Q$. It is clear now that both $a$ and $b$ induce the identity automorphism in $P / Q$; and this implies that all of $C$ induces the identity on $P / Q$ and that therefore $P(1-C) \leqq Q$. Hence $Q=P(1-C)$. For the remainder of the lemma remember that, if a commutative $p$-group is the sum of 2 cyclic groups, the order of each of its cyclic subgroups cannot exceed the maximum of the orders of the 2 summands. From Lemma 2.3, the order of $c$ in $C$ equals the order of $P(1-c)$. Since, for every $c \epsilon C$, $P(1-c)$ is cyclic by property 1 of $S$, we can apply the previous remark to yield $o(c) \leqq \max [o(a), o(b)]$.

DeFinITION. If $a$ and $b$ are in $S$, we say $a$ and $b$ are disjoint elements, or just disjoint, when $P(1-a) \cap P(1-b)=0$. 
Conollary 2.3. If $C \leqq S$ is the group generated by $a$ and $b$, and if $a$ and $b$ are disjoint elements or $o(a) \neq o(b)$, then $o(a b)=\max [o(a), o(b)]$.

Proof. Assume by symmetry that $o(a) \leqq o(b)$. By Lemma $2.4, o(a b) \leqq$ $\max [o(a), o(b)]$.

Case 1. $o(a)<o(b)$.

Then $o(a b) \leqq o(b)$. Since $C$ is also generated by $a$ and $a b, o(b) \leqq$ $\max [o(a), o(a b)]$. But $o(a)<o(b)$, so $o(b) \leqq o(a b)$. So $o(b)=o(a b)=$ $\max [o(a), o(b)]$.

Case 2. $\quad a$ and $b$ are disjoint.

If $o(a)<o(b)$, then $o(a b)=\max [o(a), o(b)]$ by Case 1. Assume therefore that $o(a)=o(b)$. Since $a$ and $b$ are disjoint, $P(1-C)=P(1-a)+$ $P(1-b)$ and hence, by Lemma 2.3, $o(P(1-C))=o(a) \cdot o(b)=o(a)^{2}$. Since $C$ is likewise generated by $a$ and $a b$, we have $P(1-C)=P(1-a)+$ $P(1-a b)$; and we deduce that $o(P(1-C))$ is a divisor of $o(a) \cdot o(b)$ by Lemma 2.3. But it follows from Lemma 2.4 that $o(a b)$ is a divisor of $\max [o(a), o(b)]=o(a)$. Hence $o(a)^{2}=o(P(1-C))$ is both a divisor and a multiple of $o(a) \cdot o(a b)$. Consequently $o(a b)=o(a)=\max [o(a), o(b)]$.

\section{Section III}

We still continue the study of norm-like pairs $P, S$, obtaining various necessary and sufficient conditions for elements of $S$ to commute.

Lemma 3.1. If, for $a \in S$ and $b \in S$, any of the following four conditions holds, then $a b=b a$ :

1. $P(1-a) \leqq F(b)$ and $P(1-b) \leqq F(a)$;

2. $P(1-a) \leqq P(1-b)$;

3. $0=P(1-a) \cap P(1-b)$;

4. there exists a $c \in S$ such that $o(a) \leqq o(c)$ and $c$ and $b$ are disjoint elements.

Proof. Assume condition 1. Then $(1-a)(1-b)=0=(1-b)(1-a)$, so $1-a-b+a b=1-b-a+b a=1-a-b+b a$; cancelling from both sides, $a b=b a$. Assume 2 . If the inequality is actually equality, then property 2 of $S$ reduces condition 2 to condition 1 . So assume the inequality is strict. By Lemma 2.3, $o(a)<o(b)$; by Corollary 2.1, o(ab) $=o(b)$. Applying Lemma 2.4, $P(1-b)=P(1-a b)$. As in the argument above, $b$ commutes with $a b$, so $a b=b a$. Assume 3 . Assume by symmetry that $o(a) \leqq o(b)$. Let $C$ be the subgroup of $S$ generated by $a$ and $b$. Then

$$
o(P(1-C))=o(a) \cdot o(b)=\frac{o(a) \cdot o(b a)}{o[P(1-a) n P(1-b a)]} .
$$

By Corollary $2.1, o(b a) \leqq o(b)$, so $0=P(1-a) \cap P(1-b a)$. Consider $x \in P(1-b)$. From property 2 of $S$,

$$
x(1-a)=x(1-b a) \epsilon P(1-a) \cap P(1-b a) ;
$$


so $x(1-a)=0$, and $P(1-b) \leqq F(a)$. Adding this to the fact that $P(1-a) \leqq F(a)$ yields $P(1-C) \leqq F(a)$. So $P(1-b a) \leqq F(a)$, and therefore, $P(1-b a) \leqq F(a) \cap F(b a) \leqq F(b)$, by applying $a^{-1}$. Hence, $P(1-a) \leqq$ $P(1-C) \leqq F(b)$, and we are again reduced to condition 1 , thus proving condition 3 implies $a b=b a$. Assume 4. If $a$ and $b$ are disjoint, we can apply 3 . Assume $a$ and $b$ are not disjoint. Since $P(1-d)$ is a cyclic $p$-group for every $d \in S$, and since $c$ and $b$ are assumed disjoint, $c$ and $a$ must be disjoint. Since it is assumed that $o(a) \leqq o(c)$, we reproduce the counting argument used above in 3 to yield $a$ and $c a$ are disjoint. Since $b$ and $a$ are not disjoint by assumption, $b$ and $c a$ are. By $3, b c=c b$, and $b c a=c a b$. So $c a b=b c a=c b a$, and $a b=b a$ again.

Comollany 3.1. If, for $a \in S$, there exist $c \in S$ and $d \in S$ such that $o(a) \leqq o(c) \leqq o(d)$ and such that $c$ and $d$ are disjoint, then $a \in Z(S)$; if $P(1-S) \leqq F(S)$, where $P(1-S)$ is the group generated by all $P(1-a)$ for $a \in S$ and $F(S)=\bigcap_{a \epsilon S} F(a)$, then $S$ is commutative.

Proof. Consider an arbitrary $b \in S$. If $b$ and $c$ are disjoint, we can apply condition 4 of Lemma 3.1 to yield $a b=b a$. If $b$ and $c$ are not disjoint, then $b$ and $d$ are, since $c$ and $d$ are disjoint, and we can apply condition 4 to the triple $a, d, b$ and obtain $a b=b a$. Since $b$ was chosen arbitrarily, $a$ is contained in the center of $S$. For the second half of the corollary, consider an arbitrary pair of elements $a$ and $b$ in $S . \quad P(1-a) \leqq P(1-S) \leqq F(S) \leqq$ $F(b)$; similarly, $P(1-b) \leqq F(a)$. Applying condition 1 of Lemma 3.1 yields $a b=b a$. $\quad a$ and $b$ were chosen arbitrarily, and the proof is complete.

LEMMA 3.2. If, for $a$ and $b$ in $S, a b=b a$, then $P\left(1-a^{2}\right) \leqq F(b)$.

Proof. Using property 2 of $S$,

$$
\begin{array}{r}
1-a b=1-a+a-a b=(1-a+a-a b) a b=(1-a) b+a(1-b) a \\
=b-1+1-a b+a^{2}-a^{2} b=1-a b+a^{2}-1+\left(1-a^{2}\right) \mathrm{b} ;
\end{array}
$$

the last equality follows from the fact that endomorphisms of a commutative group additively commute. Subtracting $1-a b$ from the first and last and transposing $a^{2}-1$, we have $1-a^{2}=\left(1-a^{2}\right) b$, and the result is established.

Coroluary 3.2. If $P$ is a p-group with $p \neq 2$, then $a \in Z(S)$ if and only if, for every $b \in S, P(1-a) \leqq F(b)$ and $P(1-b) \leqq F(a)$.

Proof. The sufficiency has been established by condition 1 of Lemma 3.1. For the necessity, let $a$ be in the center of $S$. Consider an arbitrary $b \in S$. From the previous lemma, $P\left(1-a^{2}\right) \leqq F(b)$. From property 1 of $S$, there exists an $x \in P$ such that $x(1-a)$ generates $P(1-a)$. From Lemma 2.2, $x\left(1-a^{2}\right)=2 x(1-a) \epsilon F(b)$. But 2 and $p$ are relatively prime, so $2 x(1-a)$ generates $P(1-a)$, and $P(1-a)$ is, therefore, contained in $F(b)$. By the same argument, $P(1-b) \leqq F(a)$. Since $b$ was chosen arbitrarily, we have the result. 
For Theorem 3.1 and for future use, we define $F_{0}=0, F_{1}=F(S)=$ $\bigcap_{a \epsilon S} F(a), F_{2}=$ all $x \in P$ such that $x \equiv x a \bmod F_{1}$ for every $a \epsilon S$, and, inductively, $F_{i}=$ the set of $x \in P$ such that $x \equiv x a \bmod F_{i-1}$ for every $a \in S$ $(i=1,2, \cdots) . \quad F_{2}$ is that subgroup of $P$ whose elements generate cosets $\bmod F_{1}$ which are left fixed by every $a \in S$; there is no confusion in applying an element of $S$ to $P / F_{1}$ since the modulus is $S$-invariant.

Theorem 3.1. If $P, S$ is a norm-like pair but with $p \neq 2$, and if $S$ is commutative, then $P=F_{2}$, and $(1-S)^{2}$, the set of $(1-a)(1-b)$ for all $a$ and $b$ in $S$, is 0 ; conversely, if $P, S$ is a norm-like pair and $P=F_{2}$, then $S$ is commutative.

Proof. Assume $S$ is commutative and $p \neq 2$. Consider $a \in S$. By Corollary 3.2, $P(1-a) \leqq F(b)$ for every $b \in S$. Therefore, for every $a \epsilon S$, $P(1-a) \leqq F(S)=F_{1}$. So, for every $x \in P$ and every $a \epsilon S, x \equiv x a \bmod F_{1}$, which is equivalent to $P=F_{2}$. Since, for every $x \in P$ and every $a$ and $b$ in $S, x(1-a) \epsilon F(b)$, we have $x(1-a)(1-b)=0$, or $(1-a)(1-b)$ is the 0-endomorphism of $P$ for every $a$ and $b$ in $S$. Since $P=F_{2}$ implies $P(1-S) \leqq F(S)=F_{1}$, the converse of the theorem is a direct consequence of Corollary 3.1 .

\section{Section IV}

Throughout this Section let $P, S$ be a norm-like pair but with the added assumption that the elements of $P$ have bounded order $p^{m}$, i.e., there exists a positive integer $m$ such that $p^{m} P=0$. By Lemma $2.3, S^{p^{m}}=1$, so $S$ contains elements of maximum order. In fact, every element of $S$ has maximum order in $S$ or can be written as a product of two such, for let $a \epsilon S$ be of maximum order in $S$ and consider $c \in S$. If $o(c)$ is maximal in $S$, there is nothing to prove. If $o(c)<o(a)$, then $o(a c)=o(a)$ by Corollary 2.1, and $c=a^{-1} a c$ is a product of two elements of maximum order.

Remark. If $S$ contains elements $a$ and $b$ of maximum order such that $a$ and $b$ are disjoint, then for an arbitrary $c \epsilon S$ application of Corollary 3.1 to the triple $c, b, a$ yields $c \in Z(S)$. Hence, $S$ equals its center and is consequently commutative.

LEMmA 4.1. If $S$ contains elements $a$ and $b$ of maximum order $p^{k}$ such that $a$ and $b$ are disjoint, then $F(S)=F(m)$ for every $m \in S$ of maximum order in $S$, $P / F(S)$ is cyclic of order $p^{k}$, and $P=F_{2}$.

Proof. Let $a$ and $b$ be elements of $S$ which are disjoint and have maximum order $p^{k}$. By duplicating the argument at the end of the proof of Corollary 2.1, we see that $a b$ and $b$ are disjoint and $a$ and $b a$ are disjoint. If $x \in F(a)$, $x(1-b)=x(1-a b)=0$ since $b$ and $a b$ are disjoint; so $F(a) \leqq F(b)$. Similarly, $F(b) \leqq F(a)$. So $F(b)=F(a)$. If $c \epsilon S$ is of maximum order in $S$, then $c$ and $a$, or $c$ and $b$, are disjoint since all $P(1-d)$ with $d \epsilon S$ are cyclic $p$-groups. Applying the above argument to the proper pair, say $c$ and $a$, 
we have $F(c)=F(a)$. Therefore, all $F(m)$ are equal for $m \in S$ of maximum order in $S$. Since $S$ is generated by its elements of maximum order [as noted at the beginning of this section], $F(S)$ is the intersection of all the $F(m)$ for $m \in S$ of maximum order. But all these $F(m)$ have been shown to be equal; and thus we have $F(S)=F(m)$ for every $m \in S$ of maximum order; and the first part of the lemma is established. As in Theorem 1.1, $P / F(m)$ is isomorphic to $P(1-m)$ which is cyclic and has order $o(m)$. But $F(S)=F(m)$ for $m \in S$ of maximum order, and $o(m)=p^{k}$. So $P / F(S)$ is cyclic of order $p^{k}$. For every $m \epsilon S$ of maximum order, $P(1-m) \leqq F(m)=F(S)$. Since every element of $S$ is of maximum order in $S$ or is a product of 2 such elements, Lemma 2.4 yields $P(1-S) \leqq F(S)=F_{1}$. This is precisely equivalent to $P=F_{2}$.

Remark. If $P / F(S)$ is cyclic, then there exists an $x \in P$ such that $x(1-a)$ generates $P(1-a)$ for every $a \epsilon S$. For let $x \epsilon P$ be a representative of a coset which generates $P / F(S)$. Consider $a \in S$. From property 1 of $S$, there exists a $y \in P$ such that $y(1-a)$ generates $P(1-a) . \quad y=k x+z$ for some integer $k$ and some $z \in F(S) . \quad y(1-a)=(k x+z)(1-a)=$ $k x(1-a)$ since $F(S) \leqq F(a)$. So $x(1-a)$ generates $P(1-a)$.

Theorem 4.1. If $P, S$ is a norm-like pair, $S$ is nilpotent of class 2, i.e., $S=Z_{2}(S)$.

Proof. If $S$ contains elements $a$ and $b$ of maximum order which are disjoint, then $S$ is commutative as has already been remarked. If $S$ does not contain 2 such elements, let $D=\bigcap_{m \epsilon S} P(1-m)$ with $m$ of maximum order in $S$; then we are going to show that $0<D \leqq P$, and that there exist elements $a$ and $b$ of maximum order such that $P(1-a) \cap P(1-b)=D$. For let $m \in S$ be of maximum order in $S$. We can consider the various intersections $P(1-m) \cap P(1-k)$ for every $k \epsilon S$ of maximum order. Since $P(1-m)$ is a cyclic $p$-group, and since the lattice of subgroups of a cyclic $p$-group is simply ordered and contains a finite number of elements, there exists a $j \in S$ of maximum order such that $P(1-m) \cap P(1-j)=E \leqq P(1-m) \cap P(1-k)$ for every $k \in S$ of maximum order in $S$. Therefore, $E \leqq P(1-k)$ for every $k \in S$ of maximum order, and hence $E \leqq D$. From the definition of $D$, $D \leqq P(1-m) \cap P(1-j)=E$. So $D=E$, and $m$ and $j$ are the elements whose existence we wish to establish. Let $T \leqq S$ be the set of all $a \in S$ such that $P(1-a) \leqq D ; T$ is a normal subgroup of $S$. If $o(D)=p^{i}$, and if $a \in S$ is of maximum order $p^{k}$, applying Lemma 2.3 to $S / T$ and $P / D$ yields $o(a T)=p^{k-i}$. We claim $a T$ has maximum order in $S / T$, for consider $c T^{\prime} \in S / T$. If $o(c)$ is maximal in $S$, then $o(c T)=p^{k-i}=o(a T)$. If not, then $o(a c)=o(a)$ from Corollary 2.1. Let $R \leqq S / T$ be the group generated by $a T$ and $a c T$. Applying Lemma 2.4 to $R$, we have $o(c T) \leqq \max [o(a T), o(a c T)]$, hence $\leqq p^{k-i}$. So $o(c T) \leqq o(a T)$ for every $c T \in S / T$, and $a T$ has maximum order in $S / T$. Since $P(1-a) \cap P(1-b)=D$,

$$
(P / D)(1-a T) \cap(P / D)(1-b T)=D,
$$


the 0 of $P / D$. Applying the first remark of this section to $S / T$, we see that $S / T$ is commutative. For every $t \epsilon T$ and every $m \epsilon S$ of maximum order, $P(1-t) \leqq D \leqq P(1-m)$. From Lemma 3.1, $t m=m t$ for every $t \epsilon T$ and every $m \in S$ of maximum order in $S$. Since $S$ is generated by its elements of maximum order, $T \leqq Z(S)$. Therefore, $S=Z_{2}(S)$.

Theorem 4.2. If $P, S$ is a norm-like pair, $P=F_{3}$.

Proof. If $S$ contains elements $a$ and $b$ of maximum order which are disjoint, then, by Lemma 4.1, $P=F_{2} \leqq F_{3}$; so $P=F_{3}$. If $S$ does not contain two such elements, we can define $T$ and $D$ as in the previous theorem. Since $S / T$ is an automorphism group of $P / D$ satisfying the hypothesis of Lemma 4.1, we conclude that $P / D$ equals its $F_{2}$ with respect to $S / T$. Since $D \leqq F(S)=F_{1}$, this is equivalent to $P=F_{3}$.

Theorem 4.3. If $P, S$ is a norm-like pair such that $S$ contains elements a and $b$ of maximum order which are disjoint and such that $p \neq 2$, then $S$ and $P(1-S)$ are isomorphic.

Proof. By Lemma 4.1, $P / F(S)$ is cyclic; therefore, by the second remark of this section, there exists an $x \in P$ such that $x(1-c)$ generates $P(1-c)$ for every $c \in S$. The existence of the elements $a$ and $b$ implies that $S$ is commutative; this coupled with the fact that $p \neq 2$ implies $P(1-d) \leqq F(c)$ for every $d$ and $c$ in $S$, by Theorem 2.1. Therefore, for every $c$ and $d$ in $S$ and every $y \in P$,

$$
\begin{aligned}
& y(1-c d)=y(1-c+c-c d) \\
& \quad=y(1-c)+y(1-d) c=y(1-c)+y(1-d) .
\end{aligned}
$$

If, for $a_{i} \in S$ and $y_{i} \in P, \sum_{i} y_{i}\left(1-a_{i}\right)$ is an arbitrary element of $P(1-S)$, then there exist integers $k_{i}$ such that

$$
\sum_{i} y_{i}\left(1-a_{i}\right)=\sum_{i} k_{i} x\left(1-a_{i}\right)=\sum_{i} x\left(1-a_{i}^{k_{i}}\right)=x\left(1-\prod_{i} a_{i}^{k_{i}}\right) \text {. }
$$

So every element of $P(1-S)$ equals $x(1-c)$ for some $c \epsilon S$ and that special $x$ whose existence was established earlier in the proof. If we map $c \in S$ to $x(1-c) \epsilon P$, we have a homomorphism from $S$ onto $P(1-S)$, and its kernel is exactly 1 .

\section{Section $V$}

We now turn to consideration of the norm $N(G)$ of the group $G$. By Theorem 1.1, we see that, if $P, S$ is a norm pair, then $P, S$ is a norm-like pair. We can therefore apply all results established in Sections II, III, and IV to norm pairs $P, S$. For example, where $S$ is a member of the norm pair $P, S$ and $a \in S, o(a)=o(P(1-a))$, and $F(a)$ is normal in $G$.

Theonem 5.1. The norm $N(G)$ of the group $G$ is contained in the third center $Z_{3}(G)$ of $G$. 
Proof. It follows directly from the definition of the norm that all subgroups of $N(G)$ are normal in $N(G)$ which is, therefore, commutative or hamiltonian.

Case 1. $N(G)$ contains an element of infinite order.

In this case, the norm and center of $G$ are the same (see Baer [1,3]), and the theorem is trivially true.

Case 2. $N(G)$ is commutative and contains no elements of infinite order.

Then $N(G)$ equals the direct sum of $N_{p}$, where $N_{p}$ is the $p$-component of $N(G)$, i.e., $N_{p}$ is that subgroup of the norm consisting of all $x \in N(G)$ whose order is a power of the prime $p$. Consider the prime $p . N_{p}$ is a characteristic subgroup of $N(G)$, and $N(G)$ is characteristic in $G$; so $N_{p}$ is normal in $G$, and $G$ induces a group of automorphisms $S_{p}$ on $N_{p} . N_{p}, S_{p}$ is a norm pair. $N_{p} \leqq Z(G)$, or, by Theorem $1.1, N_{p}$ has bounded order. In the latter case we can apply Theorem 4.2 to yield $N_{p}=F_{3}$. But $F_{i}$ is precisely the intersection of $P$ with the $i^{\text {th }}$ member of the ascending central series of $G$, and therefore $N_{p}$ is contained in $Z_{3}(G)$. Since $p$ was chosen arbitrarily and $N(G)$ equals the direct sum of the $N_{p}, N(G) \leqq Z_{3}(G)$, which completes Case 2 .

Case 3. $N(G)$ is hamiltonian.

By a well known theorem hamiltonian groups are the direct sum of three groups: a commutative group all of whose elements have odd order, a commutative group all of whose nonzero elements have order 2 , and the quaternions; see Zassenhaus [1]. So again the norm of $G$ is the direct sum of its $p$-components, but, different from Case $2, N_{2}$ is not commutative. $N_{2}$ is, in fact, the direct sum of the quaternions and a commutative group all of whose nonzero elements have order $2 . \quad N_{2}$ contains a unique nonzero element $w$ such that $w=2 x$ for some $x \in N_{2}$. $\quad(w)$ is therefore a characteristic subgroup of $N_{2}$. Since $N_{2}$ is normal in $G,(w)$ is normal in $G$. Adding this to the fact that $o(w)=2$ shows that $w \in Z(G)$. Baer [2] proved that when the norm of a group is hamiltonian, the following four statements must hold: $G$ contains no elements of infinite order; $G$ contains no elements whose order is divisible by 8 ; all elements of $G$ whose order is divisible by 4 are of the form $z+v$, where $v$ commutes with every element in $N_{2}$ and $z \epsilon N_{2}$ with $o(z)=4$; all elements of $G$ whose orders are not divisible by 4 commute with every element in $N_{2}$. Hence, if the commutator $[a, x]=-a-x+a+x$ for some $a \epsilon G$ and some $x \in N_{2}$ is different from $0, a=z+v$, where $v$ commutes with $N_{2}$ and $z \in N_{2}$ with $o(z)=o(x)=4$. To see this, recall the structure of $N_{2}$. Therefore, when $[a, x] \neq 0$ for $a \epsilon G$ and $x \in N_{2}$, there exists a $z \in N_{2}$ such that $[a, x]=[z, x]=w$. Since we established earlier that $w \in Z(G), N_{2} \leqq Z_{2}(G)$. We can apply the argument of Case 2 to the complement of $N_{2}$ in $N(G)$ to show that the complement is contained in $Z_{3}(G)$. Since $N(G)$ equals the direct sum of $N_{2}$ and its complement, $N(G) \leqq Z_{3}(G)$, and the proof of the theorem is complete.

Since the norm $N(G)$ as the intersection of all normalizers of all subgroups of $G$ is a normal subgroup of $G, G$ induces a group of automorphisms on $N(G)$. 
Theorem 5.2. The group of automorphisms induced by the group $G$ on its norm is nilpotent of class 2 .

Proof. Let $S$ denote the group of automorphisms induced by $G$ on $N(G)$. If $N(G)$ contains an element of infinite order, then $N(G)=Z(G)$ (see Baer $[1,3])$; then $S=1$, and there is nothing to prove. Assume that every element in the norm of $G$ has finite order.

Case 1. $N(G)$ is commutative.

Then $N(G)$ equals the direct sum of $N_{p}$. For the prime $p$, let $S_{p}=$ all $a \epsilon S$ such that $a=1$ on $N_{q}$ for every prime $q \neq p . N_{p}$ is a characteristic subgroup of $N(G)$ for every prime $p$, and $N(G)$ is characteristic in $G$; so $N_{p}$ is normal in $G$. By using Iemma 1.1, we have $S_{p}$ is precisely the group of automorphisms induced by $G$ on $N_{p} . \quad N_{p}, S_{p}$ is a norm pair and therefore, by Theorem 1.1, is a norm-like pair. If $N_{p} \leqq Z(G), S_{p}=1$. If $N_{p}$ is not contained in the center of $G$, then $N_{p}$ has bounded order by Theorem 1.1, and we can apply Theorem 4.2 to obtain $S_{p}$ is nilpotent of class 2 . Using Lemma 1.1, we see that $S$ is the direct product of its $p$-components $S_{p}$, and therefore $S$ itself must be nilpotent of class 2 .

Case 2. $N(G)$ is hamiltonian.

Recall the structure of hamiltonian groups stated in Case 3 of Theorem 5.1 to see that $N(G)$ is again the direct sum of $N_{p}$. For $p \neq 2$, we can argue as in Case 1 to obtain $S_{p}$ is nilpotent of class 2 . Let $C=$ the centralizer of $N_{2}$ in $G$. Since $N_{2}$ is a normal subgroup of $G, C$ is normal also. Let $Q$ be the quaternions. Using Baer's result on groups with hamiltonian norm cited in Case 3 of Theorem 5.1, we see that $G=Q+C . \quad C \cap Q=2 Q . \quad S_{2}$ is isomorphic to $G / C=(Q+C) / C$ is isomorphic to $Q / Q \cap C=Q / 2 Q=$ the 4-group. So $S_{2}$ is commutative. Since commutativity was not needed in the proof of Lemma 1.1, we can again apply Lemma 1.1 to obtain $S$ equals the direct product of $S_{p}$. Since $S_{p}$ is nilpotent of class 2 for $p \neq 2$, and since $S_{2}$ is commutative, $S$ is of class 2 .

We can connect and sharpen Theorems 5.1 and 5.2 with the following theorem.

Theorem 5.3. Where $N(G)$ is hamiltonian, $N(G) \leqq Z_{2}(G)$ if and only if the group of automorphisms $S$ induced on $N(G)$ by $G$ is commutative; where $N(G)$ is a commutative group containing no elements of infinite order and $2 N_{2}=0$, $N(G) \leqq Z_{2}(G)$ if and only if $S$ is commutative.

Proof. Assume $N(G)$ is hamiltonian. By Theorem 3.1, $N_{p} \leqq Z_{2}(G)$ if and only if $S_{p}$ is commutative for $p \neq 2$. For $p=2, S_{p}$ was shown to be commutative in Case 2 of Theorem 5.2, and $N_{p} \leqq Z_{2}(G)$ was shown in Case 3 of Theorem 5.1. Since $S$ equals the direct product of $S_{p}$, and $N(G)$ equals the direct sum of $N_{p}$, the first part of the theorem is established. Now assume $N(G)$ is commutative and contains no elements of infinite order and that $2 N_{2}=0 . \quad S_{2}$ thus contains elements of maximum order. If $S_{2}$ contains 2 such which are disjoint, then, by Lemma 4.1, $N_{2}=F_{2}=Z_{2}(G) \cap N_{2}$; this 
in turn implies, by Theorem 3.1, that $S_{2}$ is commutative. If $S_{2}$ does not contain 2 elements of maximum order which are disjoint, then all $N_{2}(1-a)$ are equal for $a \neq 1 \epsilon S_{2}$, since $2 N_{2}=0$. Then $N_{2}\left(1-S_{2}\right) \leqq F\left(S_{2}\right)=$ $Z(G) \cap P$. This implies, by Corollary 3.1 , that $S_{2}$ is commutative. $N_{2}\left(1-S_{2}\right) \leqq Z(G)$ is equivalent to $N_{2} \leqq Z_{2}(G)$. Therefore, in either case, $N_{2} \leqq Z_{2}(G)$, and $S_{2}$ is commutative. For $p \neq 2, N_{p} \leqq Z_{2}(G)$ if and only if $S_{p}$ is commutative, by Theorem 3.1. So again $S$ is commutative if and only if $N(G) \leqq Z_{2}(G)$.

By examining the proof just completed and by recalling that, by Baer $[1,3]$, if the norm contains an element of infinite order, then the norm equals the center, we see there is only one obstacle to the theorem: $N(G) \leqq Z_{2}(G)$ if and only if $S$ is commutative. This obstacle is the prime 2 and the restriction it imposes on the sufficiency of the desired theorem. This restriction is exemplified by Theorem 3.1 .

\section{Section VI}

Theorem 1.1 establishes that norm pairs $P, S$ are norm-like pairs and, if $S \neq 1, P$ has bounded order. A trivial example of the converse is provided by the norm-like pair $P, S$ with $S=1$. Theorem 6.1 gives sufficient conditions for norm-like pairs to be norm pairs.

Theorem 6.1. If $P, S$ is a norm-like pair such that $P$ has bounded order $p^{m}$ with $p \neq 2$ and such that $S$ contains elements of maximum order which are disjoint, then $P, S$ is a norm pair.

Proof. The problem is to construct a group $G$ such that $P \leqq N(G), P$ is normal in $G$, and $G$ induces exactly the group of automorphisms $S$ on $P$. If $S=1$, let $G=P$. If $S \neq 1, S$ contains 2 elements of maximum order which are disjoint. From Lemma 4.1 and the second remark of Section IV, there exists an $x \in P$ such that $x(1-c)$ generates $P(1-c)$ for every $c \epsilon S$. By the first remark of Section IV and Lemma 2.3, $S$ is a commutative group of bounded order, and therefore $S$ has a basis $b_{i}$. Let $G$ be the group formed by adjoining $x_{i}$ to $P$ with the following properties: $x_{i}$ induces $b_{i}$ on $P, x_{i}+x_{j}=$ $x_{j}+x_{i}$, and $p^{m} x_{i}=x\left(1-b_{i}\right) . \quad G$ contains $P$ as a normal subgroup, and induces exactly $S$ as automorphism group on $P$. Therefore, we need only show that $P \leqq N(G)$. The general element of $G$ has the form $y+a$ with $y \in P$ and $a$ a finite sum of $x_{i}$, where a given $x_{i}$ may occur more than once. If $a \epsilon G$ induces the automorphism $a$ on $P$, then $a+y=y+a+y(1-a)$ since $P$ is commutative. Since $p^{m} P=0$ and $p$ is not divisible by 2 ,

$$
\begin{aligned}
p^{m}(a+y)= & p^{m} a+p^{m} y+y(1-a)+2 y(1-a)+\cdots \\
& +\left(p^{m}-1\right) y(1-a)=p^{m} a+\left(p^{m}-1\right) p^{m} / 2 \cdot y(1-a)=p^{m} a .
\end{aligned}
$$

By Corollary 3.2,

$$
1-c d=1-c+c-c d=(1-c)+(1-d) c=(1-c)+(1-d)
$$


for every $c$ and $d$ in $S$. Therefore, if $a=x_{1}+\cdots+x_{k}$,

$$
p^{m} a=x\left(1-b_{1}\right)+\cdots+x\left(1-b_{k}\right)=x\left(1-\prod_{i} b_{i}\right),
$$

and the automorphism $a=\prod_{i} b_{i}$ since $x_{i}$ induces $b_{i}$. Since $x(1-a)$ generates $P(1-a)$ and $p^{m} a=p^{m}(a+y), P(1-a) \leqq(a+y)$. Since $a+y$ was $a$ general element of $G$, we have, for every $z \in P$ and every $w \epsilon G, z(1-w)=$ $z-w-z+w \epsilon(w)$. This is equivalent to $P \leqq N(G)$ since every element of $G$ has finite order.

\section{BiBLIOGRAPHY}

RFINHOLD BAER

1. Der Kern, eine charakteristische Untergruppe, Compositio Math., vol. 1 (1934), pp. 254-283.

2. Gruppen mit hamiltonschem Kern, Compositio Math., vol. 2 (1935), pp. 241-246.

3. Zentrum und Kern von Gruppen mit Elementen unendlicher Ordnung, Compositio Math., vol. 2 (1935), pp. 247-249.

4. Norm and Hypernorm, Publ. Math. Debrecen, vol. 4 (1956), pp. 347-350.

HaNS ZassenhaUs

1. Theory of groups, New York, 1949.

University of ILLINOIS

Urbana, Illinois

Argonne National Laboratory

LEMONT, ILLINOIS 